\title{
Contribution of airborne fine particles containing Cryptomeria japonica pollen allergens to airborne organic carbonaceous aerosols during a severe pollination episode
}

\author{
Q. Wang ${ }^{1}$, S. Nakamura ${ }^{1}$, X. Gong ${ }^{1}$, K. Kurihara ${ }^{1}$, M. Suzuki ${ }^{1}$, \\ K. Sakamoto ${ }^{1} \&$ D. Nakajima ${ }^{2}$ \\ ${ }^{1}$ Graduate School of Science \& Engineering, Saitama University, Japan \\ ${ }^{2}$ National Institute for Environmental Studies, Tsukuba, Japan
}

\begin{abstract}
Japanese cedar pollinosis has been recognized recently as a serious social problem because of its high prevalence in Japan. It is well-known that the pollen grains of Cryptomeria japonica pollen (so-called Japanese cedar pollen) usually exist as coarse particles about $30 \mu \mathrm{m}$. However, it was supposed that the major allergen Cry $\mathrm{j} 1$ (Cry j 1 particles) could be released to the atmosphere as respirable-sized particles and modified by some air pollutants during airborne transportation. Cry $\mathrm{j} 1$ particles represent major seasonal allergen sources and are suspected to cause pollen asthma. Moreover, since Cry j 1 particles mainly consist of protein materials and cytoplasm from the pollens, they should be organic carbonaceous aerosols in fine particle sizes because protein materials are also some kind of organic carbon (OC). Therefore, one of the Cry j 1 release processes in which Cry $\mathrm{j} 1$ eluted from several simulated rainfalls of various salt components have been investigated. As a result, about $60 \%$ of Cry j 1 was released in simulated rain containing $\mathrm{Ca}^{2+}$ ions. At the same time, it is important to examine the release behavior of Cry $\mathrm{j} 1$ particles and to evaluate the source contributions calculated from Cry $\mathrm{j} 1$ particles to organic carbonaceous aerosols. The aim of this study is to examine the particle size distribution of Cry j 1 and $\mathrm{OC}$ in airborne aerosols to clarify some mechanisms provoking pollen asthma and to evaluate source contributions during a severe pollination episode of FY 2005. Airborne Cry j 1 particles were collected with high volume Andersen air samplers, and Cry j 1 and $\mathrm{OC}$ (OC1-OC4) concentrations were determined by
\end{abstract}


the ELISA method and the thermal-optical carbon analyzer respectively. More than $80 \%$ of Cry j 1 existed as fine particles below $1.1 \mu \mathrm{m}$, which consisted of OC2, OC3 and OC4. Source contributions of OC derived from pollen may be averagely occupied $\sim 30 \%$ in airborne organic carbonaceous aerosols as total OC in fine particles $\left(\mathrm{PM}_{1.1}\right)$ below $1.1 \mu \mathrm{m}$ respectively. Thus, it was possible that $\mathrm{OC}$ contribution below $1.1 \mu \mathrm{m}$ is overestimated by conventional concepts of various anthropogenic and secondary formed sources because Cry j 1 particles are also below $1.1 \mu \mathrm{m}$ which are the composite OC sources released from pollen grains.

Keywords: source contribution, PM 1.1 , Japanese cedar pollen, allergen, Cry $j$ 1, pollen asthma, organic carbonaceous aerosol, organic carbon (OC).

\section{Introduction}

In Japan, the prevalence of Japanese cedar (Cryptomeria japonica) pollinosis is reported to be about $20 \%$ and it can also be said to be the national illness (Shida [1]). The prevalence is increasing especially in urban areas where its incidence is about 28\% (Bureau of Social Welfare and Public Health, Tokyo. [2]). Since the increase in the number of hay fever patients, the reduction in the age of development of symptoms, and the development of symptoms of asthmatic conditions (Maeda et al. [3]), especially in recent years, it is a big social problem as a disease, and an immediate remedy is needed.

The causative substances of Japanese cedar pollinosis are Japanese cedar pollen allergens, and there are two kinds of major allergens. One is the basic protein Cry j 1 of molecular weight $c a$. 40,000 which is localized Ubisch bodies (ca. $0.7 \mu \mathrm{m})$ attached mainly on the surface of Japanese cedar pollen $(c a .30 \mu \mathrm{m})$ (Yasueda et al. [4]). Another is called as Cry j 2 as the basic protein of molecular weight $c a$. 37,000 which is contained the starch granules and pollen lining membrane inside the pollen (Takahashi et al. [5]).

Furthermore, although it had been thought that Cryptomeria japonica pollen invaded only the human nasal cavity and mouth, and there was originally no inhalation to a lower respiratory tract, existence of the airborne suspended particulate matter containing the Cryptomeria japonica pollen allergens (Cry $\mathrm{j} 1$ particles or Cry j 2 particles) which can invade into a lower respiratory tract has now been suggested. Some researchers found and reported that respirable allergen particles are exfoliated Ubisch bodies from the pollen surface or starch granules from ruptured pollens (Kurihara et al. [6] and Wang et al. [7]). Also respirable allergen particles invade into a lower respiratory tract and develop pollen asthma. It is very important to investigate the Cryptomeria japonica pollen main part in the urban atmosphere and the airborne behavior of allergen particles, and to grasp the dynamic state, because we want to evaluate the influence of allergen particles in the development of pollinosis, pollen asthma and the modification of allergen particles with air pollutants. In addition, long range transportation phenomena of yellow sand from the East Asian continent were found during the pollen scattering season in Japan due to the global warming. Therefore, interaction between pollen and yellow sand should be of 
concern. Actually, the pollinosis was enhanced when pollen and crustal particles (Maejima et al. [8]) were inhaled at the same time.

Since Cry j 1 particles mainly consist of protein materials and cytoplasm from the pollens, they should be classified into organic carbonaceous aerosols from a viewpoint of the environmental aerosol chemistry because protein materials are also some kinds of organic carbon (OC). Moreover, most of Cry j 1 particles exist in the fine particle ranges based on our study on the size distribution of Cry j 1 (Wang et al. [7]). It is well known that OC component contained in the suspended particulate matter (SPM) of the fine particle range are mainly conventionally considered to be the particles caused from secondary formation and the anthropogenic sources. It is considered to be important how particulate matters from the natural sources take into consideration about the emission sources of the fine particles to grasp the level of the influences (contributing ratio) of the pollen allergen containing particles as a primary emission and the natural sources. Therefore, for new knowledge and a new view advocated about the emission sources of OC fine particles, especially in research of a severe pollination episode, it is necessary to grasp the OC contribution of the particles containing pollen allergen particles as a primary emission and the natural sources during a severe pollination episode.

In this paper, firstly, in order to check that the major allergen Cry $\mathrm{j} 1$ particles exist in the fine particle ranges, the collection classified by particle diameters of the airborne Cryptomeria japonica pollen of a pollination season was performed, and the size distribution of Cry $\mathrm{j} 1$ in SPM was investigated by measuring Cry $\mathrm{j} 1$ by the analyzing method using the antibody which recognizes specifically Cry $\mathrm{j}$ 1. Moreover, OC concentration in the SPM of a pollination season and Cry $\mathrm{j} 1$ concentration were measured according to particle diameter, and the $\mathrm{OC}$ concentration measurement (or contribution ratio) of the particles of the natural pollen source in the fine particles were estimated by computing and evaluating the contribution in the fine particles based on the results of OC composition measured from Cry j 1 standard samples.

\section{Experiment methods}

\subsection{Confirming one transition process of Cry $\mathbf{j} 1$ to fine particle sizes when contacted rainfall}

To confirm transition of Cry j 1 to fine particle sizes when contacted rainfall, $500 \mathrm{mg}$ pollen was mixed with various solutions of inorganic salts, ultra pure water and extraction solution. Extraction solution was usually prepared for effective elution of Cry j 1 from pollen grains (Kurihara et al. [6]). These inorganic concentrations of $\mathrm{KNO}_{3}, \mathrm{NH}_{4} \mathrm{NO}_{3}$ (secondary particles) and $\mathrm{Ca}\left(\mathrm{NO}_{3}\right)_{2}$ (derive from yellow sand) were $80,150,500$ and $1500 \mathrm{mN}$, respectively. The Cryptomeria japonica pollen solutions were made from eluted allergen contents by filtration using a cellulose acetate membrane filter (pore size is $1.2 \mu \mathrm{m}$ ). And, pollen solutions were treated after centrifuge separation $(15,000 \mathrm{rpm}, 10 \mathrm{~min})$. Supernatant liquids were picked $1 \mathrm{~mL}$, and it was exchanged to buffer solution (10 mM HEPES, $150 \mathrm{mM} \mathrm{NaCl}, 3 \mathrm{mM}$ EDTA, $0.005 \%$ surfactant P20) by a 
centrifugal filter unit (amiconultra-4, Millipore). Then, Cry j 1 concentrations were measured by the surface plasmon resonance method (Model Biacore $\mathrm{J}$ system, GE Healthcare Co. Ltd).

\subsection{Sampling sites, periods and collection of air samples}

Airborne Cryptomeria japonica pollens and allergen particles were collected at the mountain and urban areas of Saitama in Kanto area, Japan. (1) Mountain area: Air samples in three different sizes $(<1.1 \mu \mathrm{m}, 3.3-7.0 \mu \mathrm{m},>7.0 \mu \mathrm{m})$ were collected on tissue-quartz filters using high volume air samplers (Shibata Co. Ltd., AH-600, AHV) with a flow rate of $566 \mathrm{~L} / \mathrm{min}$ for the 23 hours sampling from March 29 to April 4, 2005. (2) Urban area: Air samples were collected using an AHV with a flow rate of $566 \mathrm{~L} / \mathrm{min}$ for the 23 hours sampling from March 10 to March 16, the severe pollination episode of FY2005.

\subsection{Extraction procedure of pollen allergen (Cry j 1) from air samples}

The tissue-quartz filters $(8 \mathrm{~mm} \phi)$ collected air samples were cut out and put into centrifuge tubes (Polypropylene copolymer; PPCO), and then they were filled with Cry j 1 extract $\left(0.125 \mathrm{M} \mathrm{NH}_{4} \mathrm{HCO}_{3}\right.$ solution containing $150 \mathrm{mM} \mathrm{NaCl}, 3$ mM EDTA, 0.005 wt \% Tween 20, and 10 mM HEPES buffer solution) $2 \mathrm{~mL}$ (Kurihara et al. [6]). After carrying out the standing of the solutions in 4 degrees Celsius for 24 hours, the shaking was carried out at the shaking velocity of 192 rpm at the room temperature for 1 hour (Heidolph Co. Ltd., UNIMAX 2010). Then, the centrifugation was carried out by $3000 \mathrm{rpm}$ for 30 minutes at the room temperature (Kokusan Co. Ltd., H-11NA), and the supernatant solutions were prepared as the samples for the pollen allergen Cry $\mathrm{j} 1$ determination.

\subsection{Determination of pollen allergen (Cry j 1 ) concentrations}

Cry $\mathrm{j} 1$ concentrations in the samples were quantified by an ELISA method using anti-Cry j 1 monoclonal antibody (Seikagaku Biobusiness Co. Ltd, clone 013) and peroxidase conjugated anti-Cry j 1 antibody (Seikagaku Biobusiness Co. Ltd, clone 053) which is one of the used enzyme immunoassay, so it is high sensitivity and alternative measuring. Coloring substrate is $o$-phenylenediamine, measurement absorption wavelength of a micro plate leader (Microtech Co. Ltd, MP-1000) is $492 \mathrm{~nm}$. Finally, the absorbance of each solution obtained by an ELISA method were converted into Cry $\mathrm{j} 1$ concentrations $\left(\mathrm{ng} / \mathrm{m}^{3}\right)$ in the atmosphere.

\subsection{Calculation method of OC contributing ratio to SPM derived from Cry j 1 particles}

The OC contribution ratios to SPM estimated from Cry j 1 particles was computed as the following equation (1).

$$
\text { Contribution ratio }(\%)=\frac{\text { OC conc. from Cry j } 1 \text { particles }}{\text { OC conc. from SPM }} \times 100
$$


Here, OC concentrations from Cry j 1 particles are the values of the OC concentration derived from the measurement results of Cry $\mathrm{j} 1$ concentration in the tissue-quartz filters collected air samples (i.e. SPM). OC concentrations from Cry j 1 particles can be calculated according to equation (2).

OC conc. from Cry j 1 particles $=\frac{\text { Cry j } 1 \text { conc. from } S P M \times \text { OCmass of standard sample }}{\text { Cry j } 1 \text { mass of standard sample } \times\left(\frac{\text { Cry j } 1 \text { conc. }}{\text { Pollen mass }}\right)(2)}$

In addition, OC mass of standard sample and Cry $\mathrm{j} 1$ mass of standard sample in equation (2) were obtained by measuring the concentration of OC component contained in the Cry j 1 standard samples produced from the refining Cryptomeria japonica pollen antigen Cry $\mathrm{j} 1$ of commercial elegance (the details of production of a Cry $\mathrm{j} 1$ standard sample and the analysis procedure of $\mathrm{OC}$ component are indicated in section 2.5. Moreover, Cry j 1 concentration of pollen is $10 \mathrm{ng}$ for each pollen obtained from the literature (Yasueda et al. [9]), and the pollen in mass is $4.2 \mathrm{pg}$ for each (Saito et al. [10]).

\subsection{Quantitative analysis of carbon components contained in SPM in air samples and in Cry $\mathbf{j} 1$ standard samples}

In order to obtain OC concentration in Cry j 1 standard samples refined Cryptomeria japonica pollen antigen Cry j $110 \mu \mathrm{L}$ dropped on a quartz fiber filter $(6 \mathrm{~mm} \phi)$. After it was dried (25 degrees Celsius), it was used as Cry j 1 standard samples. It measured according to the carbon components of (OC1OC4, EC1-EC3 based on their measurable temperatures) by a thermal optical carbon analyzer (Atmoslytic Co. Ltd, DRI Model 2001 OC/EC Carbon Analyzer) of IMPROVE method. The analysis conditions of this thermal optical carbon analyzer are given in table 1. In addition, OC1 are apparently volatile organic carbon below 120 degrees Celsius which can be negligible in our analytical results because pollen allergens are consisted with non-volatile protein materials.

Table 1: $\quad$ Analysis conditions of carbon concentrations of air samples.

\begin{tabular}{lcc}
\hline $\begin{array}{c}\text { Carbon } \\
\text { fraction }\end{array}$ & Flow gas & $\begin{array}{c}\text { Temperature } \\
\text { (degrees Celsius })\end{array}$ \\
\hline OC1 & $\sim 120$ \\
OC2 & $100 \% \mathrm{He}$ & $120 \sim 250$ \\
OC3 & & $250 \sim 450$ \\
OC4 & & $450 \sim 550$ \\
\hline EC1 & $98 \% \mathrm{He}$ & $\sim 550$ \\
EC2 & $2 \% \mathrm{O}_{2}$ & $700 \sim 800$ \\
EC3 & & 700 \\
\hline
\end{tabular}


Next, quantitative analysis of the carbon components in the quartz fibre filters $(6 \mathrm{~mm} \phi)$ which collected SPM in air samples carried out in the same procedure as analysis of in the Cry $\mathrm{j} 1$ standard samples. The contributing ratio was calculated by measuring the concentration of an $\mathrm{OC}$ concentration according to particle sizes in SPM.

\section{Results and discussion}

\subsection{Transition concentrations to fine particle sizes and behavior of Cry $\mathbf{j} 1$ in simulated rain solution of different cations}

Cry j 1 concentrations which transition to fine particle sizes from Cryptomeria japonica pollen grains in the simulated rain solutions of different cations $\left(\mathrm{K}^{+}\right.$, $\mathrm{NH}_{4}^{+}$and $\mathrm{Ca}^{2+}$ ) and extraction solution were shown in figure 1. In this result, higher concentrations of Cry $\mathrm{j} 1$ were eluted in $\mathrm{Ca}\left(\mathrm{NO}_{3}\right)_{2}$ simulated rain than the others.

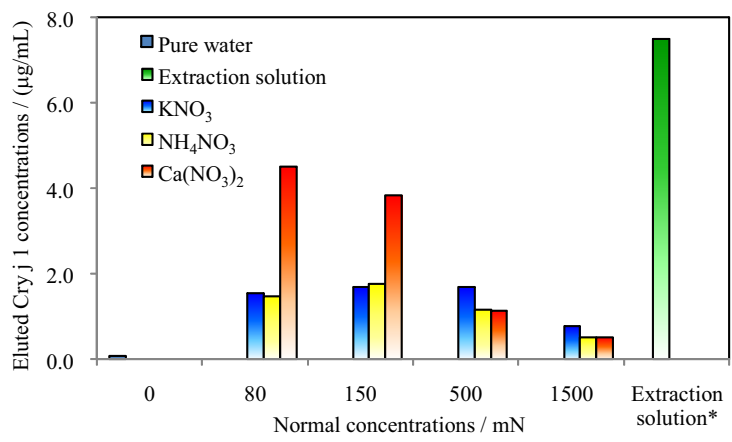

Figure 1: Tendency of eluted Cry j 1 concentrations in various normal concentrations and salt components. These samples were filtered by filter of pore size $1.2 \mu \mathrm{m}$.

*In this solution, Cry $\mathrm{j} 1$ is eluted from Cryptomeria japonica pollen grains effectively.

On the other hand, eluted concentrations were decrease when the simulated rain contained less than $80 \mathrm{mN}$ or more than $500 \mathrm{mN}$ in the concentrations. There were no differences in eluted Cry $\mathrm{j} 1$ concentrations between the normal concentrations of 80 and $150 \mathrm{mN}$ of the same salt, especially for a $\mathrm{Ca}^{2+}$ rich solution. However, they were reduced in proportion to inorganic normal concentrations between the concentrations of 150 and $1500 \mathrm{mN}$. And about $60 \%$ Cry j 1 was eluted in $\mathrm{Ca}\left(\mathrm{NO}_{3}\right)_{2}$ simulated rain compared with extraction solution. We guess that Cryptomeria japonica pollen grain has a cell membrane like a semipermeable membrane and it will be ruptured by an expansion with osmotic pressure by various ionic normal concentrations of inorganic salts in rainfall. 


\subsection{Size distribution and possible airborne behaviour of Cry $\mathbf{j} 1$ particles}

The size distribution of Cry $\mathrm{j} 1$ in different particle sizes and average airborne Cryptomeria japonica pollen counts were shown in figure 2 and Cry $\mathrm{j} 1$ concentrations in filters (below $1.1 \mu \mathrm{m}$ ) was shown in figure 3. In figure 2 , Cry $\mathrm{j}$ 1 particles were most detected at the below $1.1 \mu \mathrm{m}$ in all sampling periods. It is obvious that more than $80 \%$ of Cry j 1 concentration of all particle ranges existed in fine particles $\left(\mathrm{PM}_{1.1}\right)$ below $1.1 \mu \mathrm{m}$ which are respirable-sized particles.

The particle sizes of Cryptomeria japonica pollen is about $30 \mu \mathrm{m}$ but the Cry j 1 particles may be released to the atmosphere when Ubisch bodies are exfoliated from pollen surfaces and Cry $\mathrm{j} 1$ contents are also eluted from pollens by contacting rainfall. Therefore, Cry $\mathrm{j} 1$ content shifts to the fine particle sizes as the respirable-sized particles.

Moreover, exfoliated Ubisch bodies of Cryptomeria japonica pollen grains in an air samples were observed by a scanning electron microscope (SEM) like proving this (figure 4(a)). And ruptured pollen of content (starch granule, Golgi body, cytoplasm etc.) inside pollen which contains Cry j 1 and Cry j 2 were observed by a SEM (figure 4(b)). In our laboratory, we checked that Japanese cedar pollen allergen Cry $\mathrm{j} 1$ contents are eluted by contacting the rain water, so Cry $\mathrm{j} 1$ particles are possible to release from exfoliated Ubisch bodies of Cryptomeria japonica pollen grains, released pollen content and eluted Cry $\mathrm{j} 1$ by rain and so on. Since the major allergen Cry j 1 particles can be released to the atmosphere as respirable-sized particles, and the size distribution is even similar with the particles emitted from some anthropogenic sources such as diesel exhaust particles (DEP) (Jiang et al. [11]) and secondary formation particles, it was supposed that these pollen allergen particles may be modified by the combination and/or reaction with some air pollutants during airborne transportation.

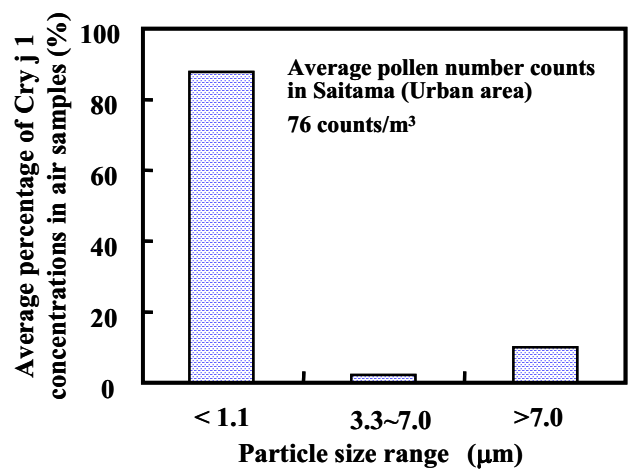

Figure 2: Average size distribution of Cry $\mathrm{j} 1$ concentrations in air samples of $<1.1,3.3-7.0,>7.0 \mu \mathrm{m}$ in Saitama (Urban area) and average airborne pollen number counts. 


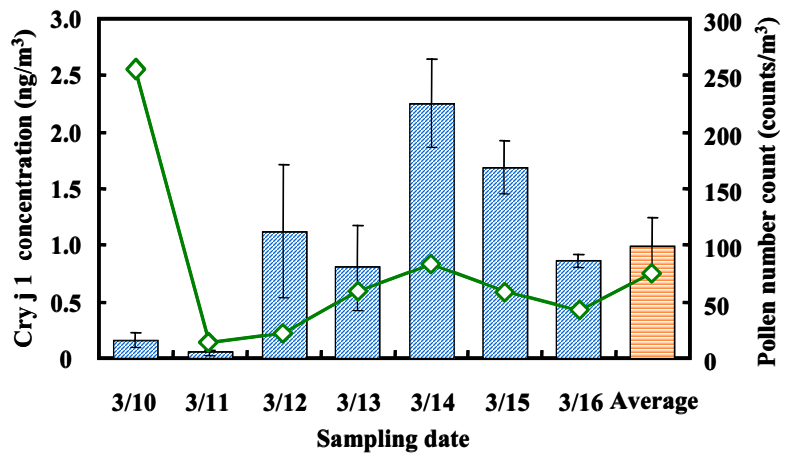

Figure 3: $\quad$ Cry j 1 concentrations in $\mathrm{PM}_{1.1}$ and Cryptomeria japonica pollen number counts for each sampling period in Saitama (Urban area).

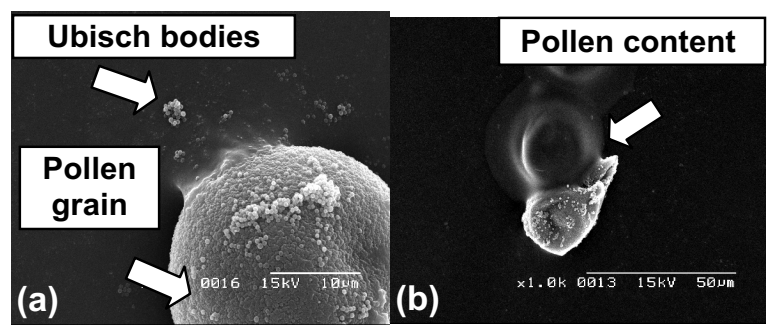

Figure 4: Scanning electron micrographs of airborne pollen. (a) Exfoliation of Ubisch body (b) Pollen rupture and release of Cryptomeria japonica pollen grains.

\subsection{Composition analysis of carbonaceous components in the Cry $\mathbf{j} 1$ standard samples}

The concentrations of the various carbonaceous components in the Cry $\mathrm{j} 1$ standard samples were shown in table 2 .

Table 2: Carbonaceous concentrations in Cry j 1 standard samples and the percentages of them.

\begin{tabular}{ccc}
\hline $\begin{array}{c}\text { Carbon } \\
\text { fraction }\end{array}$ & $\begin{array}{c}\text { Carbon concentrations } \\
\left(\mu \mathrm{g} / \mathrm{cm}^{2}\right)\end{array}$ & $\begin{array}{c}\text { Percentage of } \\
\text { carbon concentrations }(\%)\end{array}$ \\
\hline OC1 & 0.09 & 8.7 \\
OC2 & 0.08 & 7.8 \\
OC3 & 0.60 & 58.3 \\
OC4 & 0.26 & 25.2 \\
\hline EC1 & 0.00 & 0.0 \\
EC2 & 0.00 & 0.0 \\
EC3 & 0.00 & 0.0 \\
\hline
\end{tabular}


As the results of the carbonaceous measurements, we found about $58 \%$ of Cry j 1 consists of OC 3 and about $25 \%$ consists of OC4. Since its molecular weight about 40,000, it is considered to be measured in OC3 and OC4 with the high determining temperatures above 250 degree Celsius. Therefore, OC contributing ratio to SPM derived from Cry $\mathrm{j} 1$ particles is also manly computed and evaluated by the OC results of OC3 and OC4.

\subsection{Investigation of OC contribution ratios to SPM derived from Cry $\mathbf{j} 1$ particles in air samples}

The concentrations of $\mathrm{OC} 3$ and $\mathrm{OC} 4$ in particles below $1.1 \mu \mathrm{m}$ i.e. $\mathrm{PM}_{1.1}$ were shown figure 5 and figure 6 . Contribution ratios of $\mathrm{OC} 3$ and $\mathrm{OC} 4$ to $\mathrm{PM}_{1.1}$ estimated from the OC 3 and OC4 caused from Cry j 1 particles was calculated using equation (1) and equation (2). In addition, the values of (OC3 mass / Cry $\mathrm{j}$ 1 mass) and (OC4 mass / Cry j 1 mass) are $0.17 \pm 0.01,0.074 \pm 0.01$ respectively by carbonaceous analysis in the Cry $\mathrm{j} 1$ standard samples.

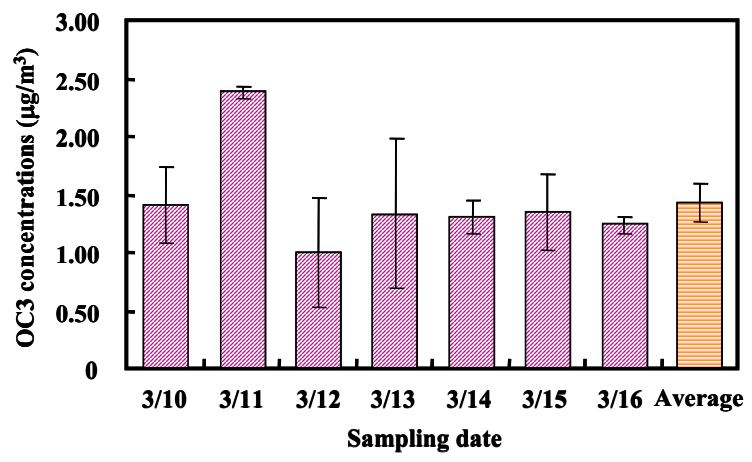

Figure 5: OC3 concentrations in air samples of $<1.1 \mu \mathrm{m}$ in Saitama (Urban area).

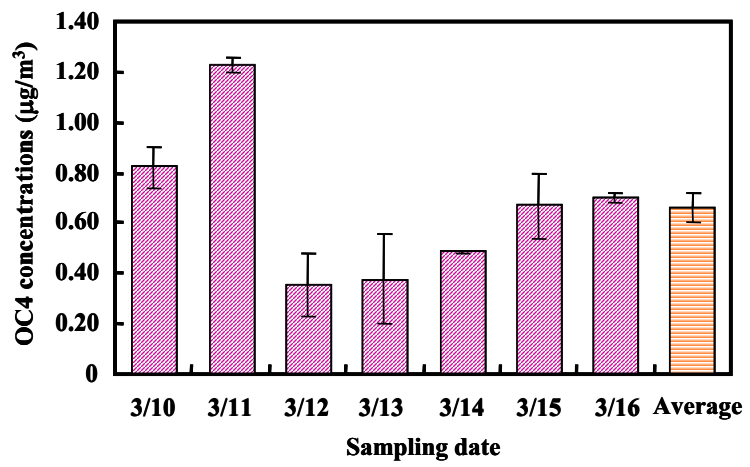

Figure 6: OC4 concentrations in air samples of $<1.1 \mu \mathrm{m}$ in Saitama (Urban area). 
The Contribution ratios to OC2, OC3 and OC4 in $\mathrm{PM}_{1.1}$ are shown in table 3 . In the severe pollination episode of FY2005, the average Contribution ratio to OC2 was $3.3 \%$, the ratio to OC3 was $13 \%$ and the ratio to OC4 was $15 \%$ in $\mathrm{PM}_{1.1}$. It is thought conventionally that the aerosols from primary emission and natural sources such as pollen have the very low $\mathrm{OC}$ contribution to $\mathrm{PM}_{1.1}$ since they distributed over the coarse particle sizes. However, Cry j 1 particles from exfoliated Ubisch bodies and eluted Cry j 1 from pollen grains were estimated that they contributed to $\mathrm{OC} 2, \mathrm{OC} 3$ and $\mathrm{OC} 4$ in the fine particle sizes. Especially, OC of Cry j 1 particles contribute to OC2, OC 3 and OC4, less volatile influence to $\mathrm{OC} 1$ and Cry $\mathrm{j} 1$ has hydrophilic and water solubility, so it is guessed the Cry $\mathrm{j}$ 1 particles may also play a role of the nuclear ambient aerosols.

Table 3: Contribution ratios of OC derived from Cry j 1 particle to ambient $\mathrm{OC}$ in $\mathrm{PM}_{1.1}$ in the sampling periods.

\begin{tabular}{|c|c|c|c|}
\hline \multirow{2}{*}{ Sampling period } & \multicolumn{3}{|c|}{ Contributing ratios* $(\%)$} \\
\hline & OC2 & OC3 & OC4 \\
\hline 3/10 & $0.30 \pm 0.10$ & $1.9 \pm 0.71$ & $1.5 \pm 0.53$ \\
\hline 3/11 & $0.050 \pm 0.017$ & $0.36 \pm 0.17$ & $0.31 \pm 0.15$ \\
\hline $3 / 12$ & $7.0 \pm 2.4$ & $19 \pm 10$ & $23 \pm 12$ \\
\hline 3/13 & $3.6 \pm 1.2$ & $10 \pm 4.8$ & $16 \pm 7.5$ \\
\hline $3 / 14$ & $7.0 \pm 2.4$ & $29 \pm 5.0$ & $34 \pm 5.9$ \\
\hline 3/15 & $3.7 \pm 1.3$ & $21 \pm 2.9$ & $19 \pm 2.5$ \\
\hline 3/16 & $1.5 \pm 0.52$ & $12 \pm 0.80$ & $9.1 \pm 0.62$ \\
\hline Average & $3.3 \pm 1.1$ & $13 \pm 2.9$ & $15 \pm 2.8$ \\
\hline
\end{tabular}

\section{Conclusion}

In this study, we confirmed that Cry $\mathrm{j} 1$ particles were transferred to fine particle sizes when contacted rainfall as the composite OC sources released from pollen grains. The air sampling of different particle sizes of the airborne pollen and allergen particles was performed during a severe pollination season of FY2005. As a result, more than $80 \%$ of Cry j 1 particles were detected as the fine particles $\left(\mathrm{PM}_{1.1}\right)$ below $1.1 \mu \mathrm{m}$, and it is clear that allergen Cry j 1 particles will release from Cryptomeria japonica pollen grains during their transportation.

Then, we measured and calculated the OC contributing ratios to SPM especially for $\mathrm{PM}_{1.1}$ derived from Cry $\mathrm{j} 1$ contents in air samples. Their contribution to ambient aerosols as the natural source particles was firstly reported. In the collection period, the average contributing ratio to OC2 was $3.3 \%$, the ratio to OC3 was $13 \%$ and the ratio to OC4 was $15 \%$ in $\mathrm{PM}_{1.1}$. Since 
the contribution ratios showed the rate of OC contents of the Cry $\mathrm{j} 1$ particles to $\mathrm{OC}$ concentration in $\mathrm{PM}_{1.1}$, it can be estimated that Cry $\mathrm{j} 1$ particles were occupied about $30 \%$ (averagely at the maximum) among total OC concentrations of $\mathrm{OC} 2, \mathrm{OC} 3$ and $\mathrm{OC} 4$ of the fine particles during a severe pollination season. However, actually, we prepared various simulated rain waters for the Cry $\mathrm{j} 1$ elution experiment, because extraction solution was not existed in natural atmosphere. Cry j 1 in simulated rain contained $\mathrm{Ca}^{2+}$ derived from yellow sand was eluted $60 \%$ compared with extraction solution. Therefore, OC contribution ratio of Cry $\mathrm{j} 1$ was possible overestimation, and this result indicated that yellow sand is one of the influence factors on release and transition of Cry j 1 to fine particle sizes. Nevertheless, it is the concentration level which cannot be negligible and the contributions have to be divided from those of secondary formation and the other anthropogenic sources.

In addition, since the concentrations were varied sharply day by day, the continuous investigation which associated the concentration of $\mathrm{OC}$ in the releasing factor of Cry $\mathrm{j} 1$ particles such as rainfall and that in the fine particles is required.

Moreover, from this study, it is obvious that Cryptomeria japonica pollen allergen Cry j 1 particles exist as fine ones below $1.1 \mu \mathrm{m}$. However, as for other pollen allergen, the possibility of existing even in the size order of nanometer is reported (Taylor et al. [12]). Some of Cry j 1 particles may also similarly exist as the particles of nanometer order. In this case, since the residence time of Cry $\mathrm{j} 1$ nano-particles in the atmosphere becomes long rather than the fine particles.

Finally, we should consider that measuring and evaluating of seasonal pollen allergen particles as their hydrophilic nucleation of ambient aerosols on the local climate effects (Ortiz et al. [13]) and their modification with the air pollutants are acquired. Therefore, it will be necessary to investigate the propagation reactions, primary emission sources of natural aerosols and the size distribution of the other seasonal pollen allergen particles in the atmosphere during their severe pollination episodes.

\section{Acknowledgements}

Some works of this study were supported by the Special Funds for Basic Research (B) (No. 17310031, FY2005-FY2007) of Grant-in-Aid for Scientific Research of Japanese Ministry of Education, Culture, Sports, Science and Technology (MEXT) and the FY2007-FY2008 Research project of Innovative Research Organization, Saitama University, Japan.

\section{References}

[1] Shida, T., Recent surveys and researches on pollinosis in Japan, Journal of Japan Air Cleaning Association, 36, pp.17-27, 1998 (in Japanese).

[2] Bureau of Social Welfare and Public Health, Tokyo Metropolitan Government, The report of the actual condition for pollinosis sufferers, pp.22-25, 2007 (in Japanese). 
[3] Maeda, Y., Pollen asthma and that treatment, Allergy in Practice, 22, pp.34-38 2002 (in Japanese).

[4] Yasueda, H., Japanese cedar pollen allergens, Journal of Clinical and Experimental Medicine, 200, pp.358-362, 2002 (in Japanese).

[5] Takahashi, Y., Trend of Japanese cedar pollen dispersal, Pollen allergy and air pollution, Shinohara Inc., pp.3-10, 2005 (in Japanese).

[6] Kurihara, K., Wang, Q., Kiryu, H., Sakamoto, K., Miwa, M. and Uchiyama, I., Airborne behavior of suspended particulate matters containing Japanese cedar pollen allergens in urban, roadside and mountain areas of Saitama, Japan, Journal of Japan Society for Atmospheric Environment, 42, pp.362-368, 2007 (in Japanese).

[7] Wang, Q., Kurihara, K., Kiryu, H., Sakamoto, K., Miwa, M. and Uchiyama, I., Daily variation differences between airborne Japanese cedar pollen and its respirable allergen particulate matters during the most severe pollination seasons, Earozoru Kenkyu, 23, pp.120-126, 2008 (in Japanese).

[8] Maejima, K., Tamura, K., Nakajima, Y., Taniguchi, Y., Saito, S. and Takenaka, H., Effects of the inhalation of diesel exhaust, Kanto loam dust, or diesel exhaust without particles on immune responses in mice exposed to Japanese cedar (Cryptomeria japonica) pollen., Inhalation Toxicology, 13, pp.1047-1063, 2001.

[9] Yasueda, H., Nitta, Y., Takatori, K., Allergen measurement technique in life environment air and research on the realities grasp, Environmental Restoration and Conservation Agency (1997-1999); Research report of (III-1-2), 1999 (in Japanese).

[10] Saito, M., Teranishi, H. and Taira, H., Study on the variation of Cry j 1 content per pollen grain among 420 Clones in 25 prefectures and estimating of heritability on Cry j 1 Sugi (Cryptometia japonica D. Don), Journal of the Japanese Forestry Society, 85, pp.312-317, 2003 (in Japanese).

[11] Jiang, Z., Wang, Q., Sekiguchi, K. and Sakamoto, K., Investigation of variations in suspended particulate matter with enforcement of regulations on diesel vehicle exhaust in suburban Japan, JSME International Journal, B, 49, pp.2-7, 2006.

[12] Taylor, P., Flagan, R., Valenta, R. and Glovsky, M., Release of allergens as respirable aerosols: A link between grass pollen and asthma, Journal of Allergy and Clinical Immunology, 109, pp.51-56, 2002.

[13] Ortiz, R., Hagino, H., Sekiguchi, K., Wang, Q. and Sakamoto, K., Ambient air measurement of six bifunctional carbonyls in a suburban area, Atmospheric Research, 82, pp.719-718, 2006. 